\title{
Construction and verification of analytical approximation of nonequilibrium neutrino distribution function in core-collapse supernova
}

\author{
Alexandra Dobrynina, ${ }^{1,2}$ Eugenia Koptyaeva ${ }^{1, *}$ and Igor Ognev ${ }^{1}$ \\ ${ }^{1}$ Department of Theoretical Physics, P. G. Demidov Yaroslavl State University, Sovietskaya 14, 150003 \\ Yaroslavl, Russia \\ ${ }^{2}$ Institute for Theoretical Physics, University of Hamburg, Luruper Chaussee 149, 22761 Hamburg, \\ Germany \\ E-mail: dobrynina@uniyar.ac.ru, koptiaeva2016@yandex.ru, \\ ognev@uniyar.ac.ru
}

The local nonequilibrium neutrino distribution function in a core-collapse supernova is considered and two variants of its analytical approximation are suggested. The proposed analytical approximations are verified using the results of a one-dimensional simulation of neutrino propagation, performed self-consistently with hydrodynamics in Prometheus-Vertex code. It was shown that the approximation, based on a Fermi-like distribution of neutrino spectrum, agrees with results of Prometheus-Vertex simulation only in the inner parts of the supernova. Whereas the approximation based on alpha-fit of neutrino energy distribution, also known as Gamma-distribution, is more general and has no restrictions for application in any part of the supernova. The obtained results could be applied for estimation of various neutrino effects in supernova conditions without their direct inclusion in the explosion simulation.

\footnotetext{
*** The European Physical Society Conference on High Energy Physics (EPS-HEP2021), ***

*** 26-30 July $2021 * * *$

*** Online conference, jointly organized by Universität Hamburg and the research center DESY ***
}

\footnotetext{
${ }^{*}$ Speaker
} 


\section{Introduction}

The theoretical investigation of a core-collapse supernova has a long history. However, the mechanisms driving explosions of massive stars at the final stage of their evolution are still open questions. Today, the main stages of this phenomenon are well known, and it is clearly that neutrino radiation plays a prominent role in the supernova explosion [1]. But numerical simulation of the neutrino radiation in supernova is a very complicated and resource-intensive problem [2]. Because of that, the inclusion in modeling of various effects of neutrino physics, which are not significant in the supernova, is difficult. Therefore, it is important to estimate the influence of such effects without including them in the explosion simulation, performed self-consistently with hydrodynamics. For these estimation it needs to know the nonequilibrium neutrino distribution function. This work is devoted to the construction and verification of two simple analytical approximations of the neutrino distribution function.

\section{Analytical approximation of neutrino distribution function}

To construct simple analytical approximations for the neutrino distribution function, we assume that their propagation in the supernova is spherically symmetric. Note that this assumption does not constrain the generality of the obtained results as one-dimensional and two-dimensional calculations lead to practically the same results [3]. This confirms that the neutrino propagation in a supernova is close to spherically symmetric. In this case, the local nonequilibrium neutrino distribution function $f_{v}$ at each point of the supernova $r$ and the time $t$ after a bounce is determined by two parameters: the neutrino energy $\omega$ and the angle $\theta$ between the neutrino momentum and radial direction of star. We also assume that the distribution function can be factorized into the angular and energy components:

$$
f_{v}(t, r, \theta, \omega) \simeq N_{v}(t, r) \Phi(t, r, \theta) F(t, r, \omega),
$$

where $N_{v}(t, r)$ is the normalized coefficient, which is determined from the neutrino number density. Note that this assumption about the factorization is quite well satisfied under supernova conditions.

Further, two variants of the analytical approximation of the neutrino distribution function are considered. The angular part $\Phi(t, r, \theta)$ for both cases is described by normal distribution (Gaussian function) of variable $y=1-\cos \theta$ as it is applicable for the most part of supernova [4]. In first variant the energy part $F(t, r, \omega)$ is approximated by Fermi-like function, suggested in Ref. [5]:

$$
f_{v}^{(F)}(y, \omega)=N_{F} \exp \left[-(y /(\sqrt{2} \sigma))^{2}\right]\left(\exp \left[\omega / T_{v}-a_{v}\right]+1\right)^{-1},
$$

where $\sigma$ is the standard deviation of the angular neutrino distribution, $T_{v}$ and $a_{v}$ are the nonequilibrium fitting spectral parameters. The second variant is based on approximation of the neutrino energy spectrum, motivated by an analytic simplicity and considered in Ref. [6], it is called " $\alpha$-fit":

$$
f_{v}^{(\gamma)}(y, \omega)=N_{\gamma} \exp \left[-(y /(\sqrt{2} \sigma))^{2}\right]\left(\omega / \omega_{1}\right)^{\gamma-3} \exp \left[-\gamma \omega / \omega_{1}\right] .
$$

where $\omega_{1}$ is the neutrino mean energy and $\gamma$ represents the amount of spectral pinching. Both variants of complete neutrino distribution function are 4-parametric. 


\section{Numerical results}

In our analysis of suggested analytical approximations we use the results of numerical simulations of explosion of SN progenitors with mass $15 M_{\odot}{ }^{1}$. The simulations were performed with the 1D version of the PROMETHEUS-VERTEX code [7]. Note that the application of concrete code does not restrict a generality of obtained results. Thus, a detail comparison of a large number of codes, calculating the one-dimensional neutrino propagation in a supernova, shows that the results obtained with them coincide in spite of significant differences in the calculation methods [8].

We can find the parameter of neutrino energy distribution function by using two first energy moments $\omega_{1}$ and $\omega_{2}$, which are defined as:

$$
\omega_{n}(t, r)=\left(\int_{0}^{\infty} F(t, r, \omega) \omega^{n+2} d \omega\right)\left(\int_{0}^{\infty} F(t, r, \omega) \omega^{2} d \omega\right)^{-1} .
$$

As analysis shown, in most part of supernova parameters of Fermi-like distribution can not be found by this method. As seen in Figure 1, in the outer part of supernova the Fermi-like approximation
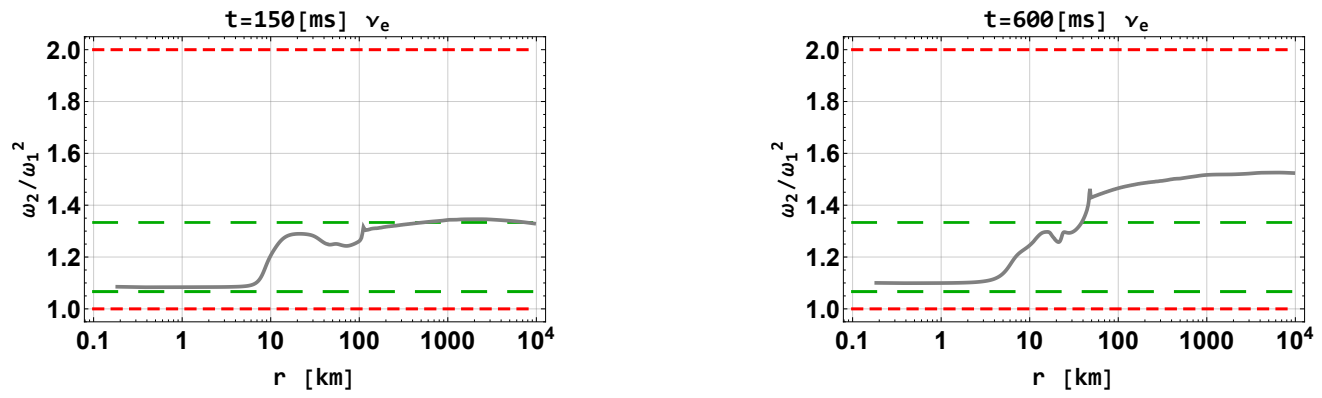

Figure 1: The ratio $\omega_{2} / \omega_{1}^{2}$ for electron neutrinos as function of the distance $r$ from the supernova center for two values of the time after a bounce $t=150 \mathrm{~ms}$ (left panel) and $t=600 \mathrm{~ms}$ (right panel). Red dashed lines correspond to upper and bottom bounds of $\omega_{2} / \omega_{1}^{2}$ for the $\alpha$-fit, green dashed lines - the Fermi-like distribution. Grey line is value of ratio $\omega_{2} / \omega_{1}^{2}$ obtained from the numerical data.

is not in agreement with first two energy moments. Moreover, their deviation from the boundary values, reachable for the Fermi-like approximation, increases with time after a bounce.

Note that this method based on first two neutrino energy moments is not the only one possible to find the parameters of the neutrino spectral distribution functions. However, the obtained results show that in the outer part of the supernova, the Fermi-like approximation certainly describes the neutrino spectrum worse than the $\alpha$-fit.

In inner part of supernova both approximations of neutrino distribution function satisfy to two first neutrino energy moments $\omega_{1}$ and $\omega_{2}$. As shown on Figure 2, the Fermi-like approximation more better coincides with numerical data than $\alpha$-fit. However, the $\alpha$-fit differs from data only in lowenergy part of spectra, which contain small fraction of all neutrino. Moreover, this deviation quickly disappears with an increase of distance from supernova center. Note, that in this region of supernova the neutrino gas is close to local equilibrium with the matter. Therefore, the approximation of neutrino gas by Fermi-like distribution is physically more justified than an application of the $\alpha$-fit.

\footnotetext{
${ }^{1}$ https://wwwmpa.mpa-garching.mpg.de/ccsnarchive/archive.html
} 

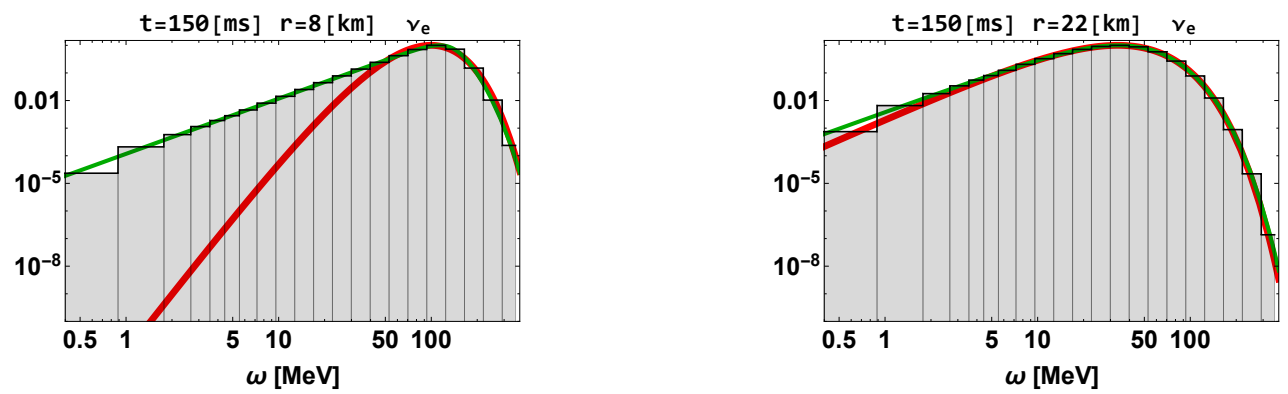

Figure 2: Spectrum of electron neutrinos, which propagate in radial direction, normalized to one, as function of neutrino energy for fixed value of time after a bounce $t=150 \mathrm{~ms}$ and two values of distance from supernova center $r=8 \mathrm{~km}$ (left panel) and $r=22 \mathrm{~km}$ (right panel). Red lines correspond to the $\alpha$-fit, green lines - the Fermi-like distribution. The numerical data is presented as grey step function.

\section{Conclusions}

Based on general assumptions about the form of the local nonequilibrium neutrino distribution function in a supernova, two variants of its analytical approximation are proposed. Using the results of one-dimensional simulation of a supernova explosion performed with the PROMETHEUSVERTEX code, the proposed analytical approximations are verified.

It is shown that the approximation, based on the Fermi-like distribution, does not correspond to the first two energy moments of the neutrino distribution function in the outer part of the supernova. Thus, the $\alpha$-fit approximation of the neutrino spectrum is more general. However, in the inner part of the supernova, the approximation based on the Fermi-like function is physically more justified and precise.

\section{Acknowledgments}

The work is supported by the RSF (Grant No. 18-72-10070). We are thankful to H.-T. Janka and his collaborators for providing us with the data of the supernova explosions and for helpful discussions. A.D. acknowledges the support by the Alexander von Humboldt foundation.

\section{References}

[1] S. A. Colgate and R. H. White, Astrophys. J. 143 (1966) 626.

[2] R. Glas, O. Just, H. T. Janka and M. Obergaulinger, Astrophys. J. 873 (2019) 45.

[3] O. Just, R. Bollig, H. T. Janka et al., Mon. Not. Roy. Astron. Soc. 481 (2018) 4786.

[4] A. Dobrynina, E. Koptyaeva and I. Ognev, J. Phys. Conf. Ser. 1690 (2020) 012003.

[5] H. T. Janka and W. Hillebrandt, A\&A 224 (1989) 49.

[6] M. T. Keil, G. G. Raffelt and H. T. Janka, Astrophys. J. 590 (2003) 971.

[7] L. Hüdepohl, PhD thesis, Technische Univ. München (2014).

[8] E. O'Connor, R. Bollig, A. Burrows et al., J. Phys. G 45 (2018) 104001. 